\title{
ORGANIZACIONES SOCIALES DEL HÁBITATY SU INFLUENCIA EN LA FORMACIÓN DE LAS LEYES Y LAS POLIIIICAS SOCIALES
}

THE SOCIAL ORGANIZATIONS OF THE HABITAT AND THEIR INFLUENCE ON THE FORMATION OF LAWS AND SOCIAL POLICIES

\section{Florencia Bareiro Gardenal ${ }^{2}$}

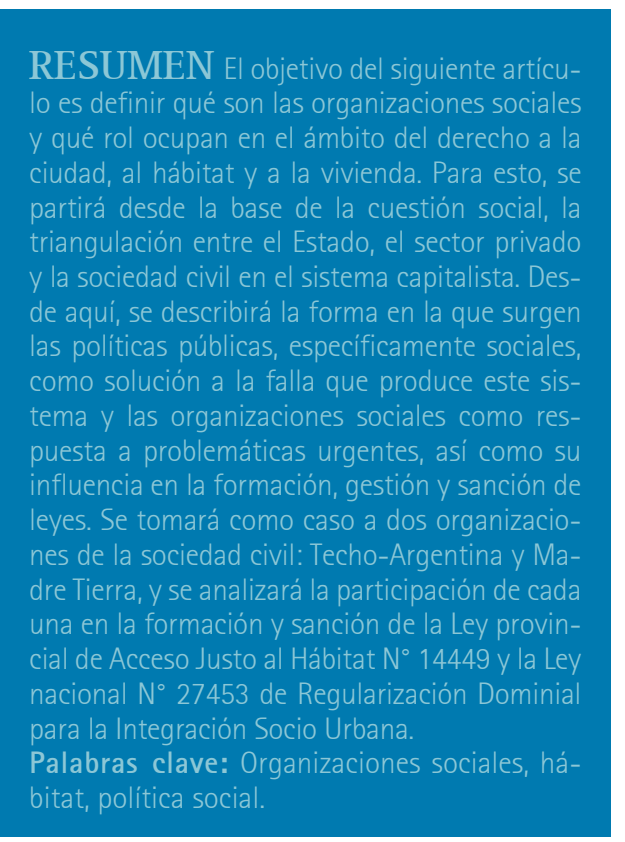

1 Recibido 2 de mayo de 2019. Aceptado 11 de octubre de 2019.

Parte de lo presentado en este artículo ha sido desarrollado en el trabajo final de grado para la Licenciatura en Comunicación Social de la Universidad Nacional de La Matanza.

${ }^{2}$ Licenciada en Comunicación Social de la Universidad Nacional de La Matanza. Correo electrónico: bareirogardenal@gmail.com.

\section{LA CUESTIÓN SOCIAL Y LAS ORGANIZACIONES SOCIALES DEL HÁBITAT}

En el modo de producción capitalista, el capital conforma la relación social hegemónica y tiene como fundamento de su constitución un proceso de desigualdad material entre los poseedores de los medios de producción y los no poseedores (Chahbenderian, 2018). Al retomar la teoria de Marx, la forma capitalista de la extracción de plusvalor presupone la igualdad jurídica de los miembros de la sociedad. La explotación se basa en la compraventa de la fuerza de trabajo. Así, quienes tienen como único medio para garantizar su subsistencia la venta de su fuerza de trabajo en el mercado, pueden no encontrar una demanda efectiva o recibir a cambio un salario que no sea suficiente para asegurar la reproducción de su vida.

Pero, además, el capital debe asegurarse que la fuerza de trabajo que no emplea hoy permanezca disponible en caso de ser requerida en un futuro. Entonces, el Estado, como representante inmediato del capital total de la sociedad, es el que lo garantiza a través de las políticas públicas, en general, y las sociales, en particular, que sirven para asegurar la reproducción obrera si esta peligra debido a las dificultades de garantizarla vía mercado laboral (De Sena, 2011). El Estado, como forma necesaria del capital, es el explotador de la clase obrera como tal, pues es el garante de que la misma produzca plusvalía en condiciones normales asegurando los máximos posibles de "homogeneidad" entre integración social e integración sistémica. Por ello, en las sociedades capitalistas, el problema de la integración social se ubica en el centro de las discusiones respecto al orden social.

De esta manera, se interpreta que, frente a la incapacidad del sistema para asegurar la reproducción de cada una de las personas, se requiere la aplicación de políticas sociales que acompañen o potencien este sistema y puedan reparar esa falla. Las politicas sociales resultan eficaces para menguar la lucha de clases y disminuir los costes laborales a cargo de los empleadores, al mismo tiempo se financian con la plusvalía arrancada a otras fracciones de la sociedad, a los sectores más vulnerables. Por 
De Prácticas y discursos

Universidad Nacional del Nordeste

Centro de Estudios Sociales

Año 8, Número 12, 2019, Octubre

ISSN 2250-6942

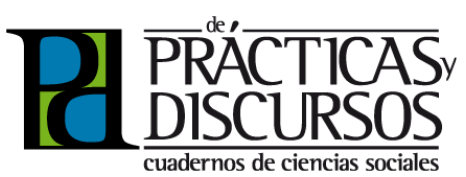

ABSTRACT The objective of the following article is to define what social organizations are and what role they play in the field of the right to the city, to the habitat and to housing. For this, we will start from the base of the social question, the triangulation between the State, the private sector and civil society in the capitalist system. From here, we will describe the way in which public policies, specifically social policies, arise as a solution to the failure that this system produces and the role of social organizations in response to urgent problems, as well as their influence on training, management and enactment of laws. Two civil society organizations will be taken as a case: TECHO-Argentina and Madre tierra and each one will be analyzed for the formation and sanction of the Provincial Law of Fair Access to Habitat 14,449 and the national Law 27,453 of Regularization of Land for Urban Social Integration

Keywords: Social organizations, habitat, social policy. ende, la razón de ser de las políticas sociales consiste en asegurarse que "la mayoria de la población acepte esta dominación del capital" (Gough, 2003; Halperin Weisburd, 2008, citado en De Sena, 2011). En este panorama aparece la cuestión social, en el siglo XIX, cuando se vislumbra el riesgo de destrucción de la sociedad liberal, debido a las nuevas tensiones generadas por la industrialización. "Así emerge 'lo social', entendido como los sistemas de regulación (diferentes a los del mercado), instituidos para intentar llenar la brecha entre lo político y lo económico" (Hopp, 2010: 4, citado en De Sena, 2011).

La cuestión social, en el contexto del Estado moderno constituido en el proceso de acumulación capitalista que se funda en la desigualdad estructural y la dependencia que resulta de la subordinación del trabajo al capital, hace alusión a tres tipos de "fallas", según Scribano y De Sena (2014): Ias del mercado, las del Estado y las de la sociedad civil. Los procesos de desigualdad y expulsión generados en la estructuración de una sociedad basada en la mercantilización de la vida provocan quiebres conflictuales que deben ser subsanados sistémicamente. Si los mecanismos de solidaridad, cohesión, ayuda y organización social eran antes concebidos y visualizados como "naturales", con el desarrollo de la modernidad se constituyen en dispositivos artificiales, centrados especialmente en una nueva noción de individuo, donde este se transformará en el culpable o responsable individual de sus padecimientos y, a su vez, en generador de la fractura de la sociedad (Carbadella, citado en Scribano y De Sena, 2014).

Grassi (2000) afirma que hay dos elementos que hacen a la cuestión social en el capitalismo y que interpelan la legitimidad del Estado moderno: la cuestión de la pobreza, en tanto refiere a las condiciones de reproducción de la vida; es decir, a las limitaciones en el acceso a bienes y servicios y en la participación e integración socio-cultural; y la cuestión del trabajo, entendida como capacidad humana que, organizada por el mercado, viabiliza y legitima el acceso a los recursos, la protección y la seguridad de los sujetos y las condiciones de su participación en la vida sociocultural. El paso de los años y la historia del mundo laboral e industrial fue modelando la cuestión social en la Argentina, diluyéndose las sociedades de beneficencia como tal, apareciendo el Estado con preponderancia en la cuestión social, pero también, a partir del siglo XX, crecieron las denominadas organizaciones de la sociedad civil, con alta participación en el diseño e implemen- 
De Prácticas y discursos

Universidad Nacional del Nordeste

Centro de Estudios Sociales

Año 8, Número 12, 2019, 0ctubre

ISSN 2250-6942
Organizaciones sociales del hábitat y su influencia

en la formaciōn de las leyes y las políticas sociales

The social organizations of the habitat and their influence on the formation of laws and social policies

tación de las políticas sociales, dejando al menos el interrogante respecto a la cuestión de la pobreza como un problema social que admite la posibilidad de acciones de asistencia estatal o de beneficencia privada.

Siguiendo a De Sena (2011), la denominada "cuestión social" acompañó al desarrollo del capitalismo desde sus orígenes, como "efecto" y "causa" de sus procesos de gestación y desarrollo. Así, los cambios y transformaciones en todos los campos de la vida social que esta implicaba y representaba, las intervenciones sociales del Estado a que dieron lugar (las políticas sociales, entre otras) fueron (y son) objeto de debate (y reflexión) entre y de distintas miradas y posturas teóricas y políticas. Históricamente, el modo de abordar la cuestión social fue a través de las políticas sociales como intervenciones estatales en y sobre la sociedad, las que abarcaban no solo políticas de empleo, sino también políticas más extensivas que "atendian" problemas de infraestructura, vivienda, salud, educación.

Di Virgilio (2012) describe que a mediados de 1990 un importante número de organizaciones de diferentes tipos, organizaciones sociales, se involucró en la gestión del hábitat de interés social. Estas experiencias se orientan hacia la construcción de vivienda, el reordenamiento de villas, la provisión de lotes con servicio, la construcción de redes de agua y cloacas, la capacitación y el asesoramiento constructivo, la regularización dominial, entre otras cosas. Estas organizaciones sociales fueron contempladas por algunos programas sociales del área metropolitana de Buenos Aires para que actúen como organismos responsables de su implementación. En relación con el rol que comenzaron a tener las organizaciones de la sociedad civil (OSC), De Piero (2005) señala que se instala un discurso que les otorga a estas una cantidad importante de virtudes y de supuestos respecto de las políticas públicas, de algunas de las cuales el Estado estaría careciendo y se agrupan en cinco puntos centrales. En primer lugar, la transparencia, porque los miembros de las OSC tienden a ser menos opacos en el manejo de fondos. La cercanía con los beneficiarios que es posibilitado por el anclaje territorial que les brinda un conocimiento de la realidad local que el Estado no posee con la misma claridad. La operatividad, al lograr con mayor eficacia y eficiencia sus prácticas. Su mayor flexibilidad y la baja de costos al sostenerse en el voluntariado. Así como De Piero (2005) señala algunas virtudes que se les otorga a las OSC, Maglieri (2003) desarrolla algunos de los prin- 
De Prácticas y discursos

Universidad Nacional del Nordeste

Centro de Estudios Sociales

Año 8, Número 12, 2019, Octubre

ISSN 2250-6942

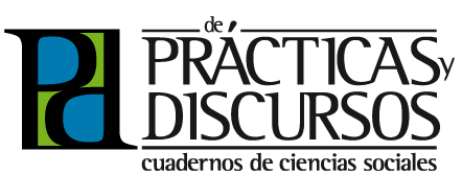

cipales problemas que enfrentan las organizaciones sociales, como escasez de financiamiento o de recursos, el aumento de la competitividad dentro y fuera del sector, la presión de la sociedad por visualizar resultados y transparencia en la gestión, el reclutamiento de socios y la capacidad de poder diferenciarse para lograr un posicionamiento más fuerte. A las organizaciones sociales les toca asumir un rol protagónico. Deben adaptarse, crecer, mejorar y desenvolverse para ocupar ese lugar semivacio, reclamado explícito o implícitamente por la sociedad y en muchos casos dejado por el Estado, que se ha creado por las nuevas estructuras socioeconómicas de los años actuales y su proyección futura. La sociedad necesita contar con un sector social eficiente y efectivo, con capacidad de desarrollo acorde con las necesidades actuales, y que acepte la creciente tendencia de su protagonismo.

Di Virgilio (2012) señala algunas experiencias de micro-financiamiento habitacional, como intervenciones compartidas, donde las organizaciones sociales se constituyen en ámbitos a través de los cuales es posible acceder a los beneficios de los programas orientados a la producción social del hábitat. El acceso al beneficio está fundamentalmente mediado por las capacidades de gestión que hayan adquirido las organizaciones a lo largo del proceso de constitución. De este modo, las OSC, a través de sus intervenciones, también se incorporan a las redes de las familias de menores ingresos. Las organizaciones sociales centraron sus actividades en la promoción y la administración de proyectos, y en la asistencia técnica y social a la población beneficiaria, articulando para ello con organizaciones de base, universidades y agencias de cooperación.

Entonces, en el caso de las organizaciones sociales relacionadas al hábitat, tierra y vivienda, en la Argentina se puede observar que tienen una tendencia a la promoción y el desarrollo. Como destaca De Piero (2005) sobre esta categoría, se trata de organizaciones que no buscaron exclusivamente la satisfacción de demandas materiales por medio de la asistencia directa, sino también la articulación en el interior de las comunidades, urbanas o rurales; la mediación con el Estado, la promoción humana vinculada a la educación, protección de la salud, estímulo a las actividades culturales, etc. En el seno de esta amplia corriente se encuentran distintas opciones metodológicas, áreas de trabajo y variadas posturas en las discusiones políticas que se plantean en su interior. 
De Prácticas y discursos

Universidad Nacional del Nordeste

Centro de Estudios Sociales

Año 8, Número 12, 2019, 0ctubre

ISSN 2250-6942
Organizaciones sociales del hábitat y su influencia

en la formación de las leyes y las políticas sociales

The social organizations of the habitat and their influence on the formation of laws and social policies

Las organizaciones de desarrollo y de promoción son un típico producto de las sociedades emergentes o periféricas. Donde el Estado no es capaz de cubrir, territorial y temáticamente, todas las demandas sociales y donde el mercado, que en sí mismo no genera inclusión y provoca desigualdad, no estimula ciclos productivos inclusivos, con una burguesía "nacional" muy débil, o directamente en condiciones de economías de enclave. La pobreza, la marginación y el subdesarrollo han sido situaciones identitarias para América Latina desde los tiempos de la organización de los diferentes Estados. Si en Europa la cuestión fue generar una sociedad civil capaz de resistir el poder de las monarquías absolutas, o el resto de los poderes no representativos (la Iglesia, el señor feudal, la nobleza), para América Latina consistió básicamente en crear espacios de participación a favor y en defensa de los derechos básicos a lo largo de su historia, ya que ellos fueron, y son, negados a buena parte de la población. (De Piero, 2005: 143)

En el siglo XXI, siguiendo a Ortiz Flores (2010), el contexto global y regional se enfrenta a cuatro situaciones derivadas del proceso de globalización económica que está conducido por los intereses transnacionales, el capital financiero y sus aliados en los gobiernos y los medios de comunicación. Una de ellas es el empobrecimiento creciente de grandes masas de la población mundial, acompañado del despojo y destrucción de sus recursos: económicos, naturales y culturales. Otra de las situaciones es la exclusión en diferentes formas. Económica: la desposesión y la pobreza extrema. Social: la negación de los derechos económicos (al trabajo digno y bien remunerado) y sociales (a la alimentación, la salud, la vivienda y la educación de calidad). Política: la imposibilidad de incidir en las decisiones; la imposibilidad de participar y de ejercer los derechos ciudadanos. Cultural: la homogeneización impuesta como proyecto de dominación y mercadeo que destruye las culturas y formas de vida locales. Psicológica: la pérdida de la autoestima. La expropiación de los haberes y saberes de los pueblos es la tercera situación. De sus excedentes económicos y de los recursos existentes en sus territorios: el agua, la biodiversidad, los bosques y los recursos minerales y energéticos. Así como de sus conocimientos y habilidades tecnológicas. La cuarta situación es la destrucción de los colectivos mediante normas y prácticas que individualizan los problemas y 
De Prácticas y discursos

Universidad Nacional del Nordeste

Centro de Estudios Sociales

Año 8, Número 12, 2019, Octubre

ISSN 2250-6942

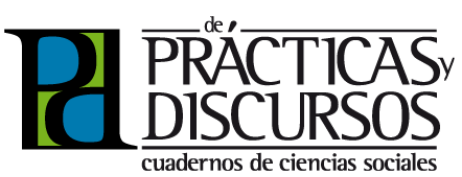

las soluciones, dividen las comunidades, destruyen las prácticas comunitarias, atacan y buscan subordinar a los sindicatos, organizaciones sociales, cooperativas y movimientos sociales a los intereses económicos y políticos de los poderosos.

Ante las tendencias, impactos y retos que genera la globalización económica, Ortiz Flores (2010) señala que son muchos los colectivos (redes, movimientos sociales, foros permanentes) que se plantean, tanto a escala local como nacional o internacional, la necesidad urgente de poner en marcha experiencias transformadoras capaces de cimentar un cambio profundo que contribuya a construir un mundo para todos. Se vienen impulsando en la región latinoamericana tres temas que se articulan entre si para abrir nuevos cauces transformadores a la producción, gestión, uso y disfrute del hábitat humano: el derecho a la ciudad, la producción social del hábitat y la gestión participativa del hábitat. El derecho a la ciudad es un derecho colectivo que persigue como objetivo una ciudad incluyente, solidaria, equitativa, participativa, productiva, sustentable, habitable y disfrutable para todos.

\section{LAS LEYES EN ARGENTINA QUE PROMUEVEN POLITICAS SOCIALES DE HÁBITAT}

En este marco es posible encontrar en la Argentina dos leyes que se formaron sobre la base del derecho a la ciudad con la participación de organizaciones de la sociedad civil. Una a escala provincial en Buenos Aires es la Ley 14449 de Acceso Justo al Hábitat (LAJH) que se aprobó en 2012. El principal objeto de la ley es la promoción del derecho a la vivienda y a un hábitat digno y sustentable en la provincia de Buenos Aires. Sus objetivos específicos son producir suelo urbano, facilitar la regularización urbano dominial de barrios informales, atender de manera integral la diversidad y complejidad de la demanda urbano habitacional, y generar nuevos recursos a través de instrumentos que permitan, al mismo tiempo, reducir las expectativas especulativas de valorización del suelo. En consonancia con estos objetivos, la norma fija cuatro principios rectores que son su fundamento jurídico. Ellos son: el derecho a la ciudad y a la vivienda, la función social de la propiedad, la gestión democrática de la ciudad y el reparto equitativo de cargas y beneficios.

La Constitución nacional considera el derecho a la vivienda digna en el artículo 14 bis y, a partir de la incorporación de tratados 
y convenciones de Derechos Humanos a la Constitución en 1994, este derecho se ha ampliado y complejizado. El sistema universal de protección de Derechos Humanos reconoce el derecho a una vivienda adecuada (artículo 11 del Pacto Internacional de Derechos Económicos, Sociales y Culturales, Pidesc) como: disponer de un lugar donde poderse aislar si se desea, espacio adecuado, seguridad adecuada, iluminación y ventilación adecuadas, una infraestructura básica adecuada y una situación adecuada en relación con el trabajo y los servicios básicos, todo ello a un costo razonable (Observación general $N^{\circ} 4$ del Pidesc).

La ley reconoce al déficit habitacional como un problema multidimensional: no se trata solamente de que la cantidad de unidades de vivienda sea insuficiente para la población, sino de que existe un déficit urbano integral, que es un límite para el ejercicio de otros Derechos Humanos, como el acceso a la educación, a la salud y al trabajo, entre otros. Entonces, siguiendo a Bustos (2015), para cumplir con el objetivo de que todos puedan acceder a un hábitat digno, la ley crea una serie de instrumentos financieros impositivos, de gestión de suelo, de planificación urbana y de redistribución de la renta, como:

El fomento al crédito para el mejoramiento y ampliación de viviendas, a través de un Fondo Fiduciario Provincial. La generación de lotes con servicios. La integración socio urbana de villas y asentamientos, para lo cual se crean mesas de gestión con participación de los sectores involucrados en cada proyecto de urbanización. El establecimiento de zonas de Promoción de Hábitat Social, que deberán determinar los municipios en sus planes. Participación de los municipios en las valorizaciones inmobiliarias generadas por la acción del Estado (obras, cambios de zonificación, cambios de indicadores urbanísticos, aprobación de grandes desarrollos inmobiliarios). Impuesto progresivo al baldio en casos de declaración de interés prioritario de áreas por parte de los municipios y luego de un plazo en el cual el propietario no cumpla con la obligación de parcelar o edificar. Consorcios urbanísticos que permiten asociaciones público-privadas para nuevos loteos $y$ planes de vivienda.

La LAJH define y especifica para el ámbito de la provincia de Buenos Aires el derecho reconocido por la Constitución nacional a una vivienda digna: "Todos los habitantes de la Provincia 
De Prácticas y discursos

Universidad Nacional del Nordeste

Centro de Estudios Sociales

Año 8, Número 12, 2019, Octubre

ISSN 2250-6942

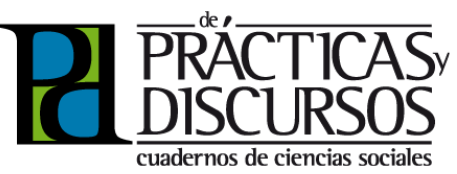

tienen garantizado el derecho al uso y goce de la ciudad y de la vivienda" (art. 11). Siguiendo al documento del CELS (2017), este enfoque ha sido sustento de decisiones judiciales que ordenaron al Poder Ejecutivo provincial satisfacer las demandas habitacionales urgentes. Al incorporar la noción de que el acceso a la vivienda es también una "forma de favorecer el ejercicio pleno de los derechos fundamentales", se lo considera como un derecho indivisible e interdependiente de los Derechos Humanos. La LAHJ promueve la toma de decisiones con pleno protagonismo de la comunidad y sus organizaciones, que fomentan el acceso al hábitat y la vivienda (art. 13). Impulsa la institucionalización de canales de participación y consenso. Reconoce la autogestión de las organizaciones sociales como un atributo de la gestión democrática de la ciudad.

Por otro lado, en 2015, con la asunción del nuevo gobierno nacional de la Alianza Cambiemos, se generó el escenario político para que un conjunto de organizaciones sociales y no gubernamentales plantee a las nuevas autoridades la necesidad de avanzar en la formulación e implementación de una política de integración urbana para los barrios populares informales. La mesa de negociaciones estuvo integrada, entre otros, por la Confederación de Trabajadores de la Economía Popular (CTEP), la Corriente Clasista y Combativa (CCC), Barrios de Pie (BdP), Un Techo para mi País (Techo) y el vicejefe de Gabinete de Ministros, Mario Quintana. Fruto de esto, durante 2016 se consensuó la propuesta de realizar un primer relevamiento de barrios populares. Se creó con esa finalidad la Unidad Ejecutora de Proyectos Socio Comunitarios dependiente de la Secretaría de Coordinación Interministerial de la Jefatura de Gabinete de Ministros de la Nación. La metodología para llevarlo a cabo fue aportada por Techo, que venía realizando un relevamiento similar en el país desde 2012 (Guevara, Marigo y Wallace, 2018).

El Registro Nacional de Barrios Populares en Proceso de Integración Urbana (Renabap), incorporado dentro de la Agencia de Administración de Bienes del Estado (Aabe), tiene como objetivo registrar los bienes inmuebles donde se asientan los barrios populares y las construcciones existentes en dichos barrios al 31 de diciembre de 2016 y obtener datos de las personas que los habitan a efectos de desarrollar políticas públicas habitacionales inclusivas. Sobre la base del registro se deberán realizar las políticas de regularización dominial enmarcadas en la Ley $N^{\circ}$ 
De Prácticas y discursos

Universidad Nacional del Nordeste

Centro de Estudios Sociales

Año 8, Número 12, 2019, 0ctubre

ISSN 2250-6942
Organizaciones sociales del hábitat y su influencia

en la formaciōn de las leyes y las políticas sociales

The social organizations of the habitat and their influence on the formation of laws and social policies

23967, a partir de lo cual la Aabe estará facultada para suscribir convenios con provincias, municipios y privados a los fines de posibilitar la transferencia de los inmuebles a las familias ocupantes. Asimismo, la agencia queda facultada para entregar -en conjunto con la Anses- Certificados de Vivienda Familiar a los fines de acreditar la existencia y veracidad del domicilio, a los efectos de solicitar la conexión de servicios tales como agua corriente, energía eléctrica, gas y cloacas; solicitar la Clave Única de Identificación Tributaria (Cuit) y/o Laboral (Cuil), realizar peticiones ante los organismos públicos; solicitar prestaciones de salud, previsionales y educativas.

En el texto de la Ley 27453 de Regularización Dominial para la Integración Socio Urbana (2018) se explica que se entiende por integración socio urbana al conjunto de acciones orientadas a la mejora y ampliación del equipamiento social y de la infraestructura, el acceso a los servicios, el tratamiento de los espacios libres y públicos, la eliminación de barreras urbanas, la mejora en la accesibilidad y conectividad, el saneamiento y mitigación ambiental, el fortalecimiento de las actividades económicas familiares, el redimensionamiento parcelario, la seguridad en la tenencia y la regularización dominial. Tales acciones deberán ser progresivas, integrales, participativas y con enfoque de género y diversidad. Además, es de destacar el artículo 15 de esta ley que hace referencia a la suspensión, por el plazo de cuatro años, de las acciones y medidas procesales que conduzcan al desalojo de los bienes inmuebles incluidos en el Renabap, tanto los sujetos a expropiación como aquellos de propiedad del Estado nacional. Discusiones al interior de las leyes y la participación de las OSC Para entender el rol de las organizaciones sociales en el hábitat dentro de las políticas públicas, se llevó a cabo desde un abordaje cualitativo una serie de entrevistas a miembros de dos organizaciones que tuvieron un rol protagonista en la creación de las leyes. Por un lado, Madre Tierra fue una de las organizaciones impulsoras de la Ley de Acceso Justo al Hábitat 14449 en la provincia de Buenos Ares. Por otro, Techo-Argentina aportó la metodología que venía utilizando en sus propios relevamientos de barrios populares para crear el Renabap y sobre la base de los datos que se pudieron obtener se gestó la Ley de Regularización Dominial 27453 en conjunto con otros actores de la sociedad.

Para realizar una breve descripción de las organizaciones, se llevó a cabo una etnografía virtual, la cual permite el estudio de 
De Prácticas y discursos

Universidad Nacional del Nordeste

Centro de Estudios Sociales

Año 8, Número 12, 2019, Octubre

ISSN 2250-6942

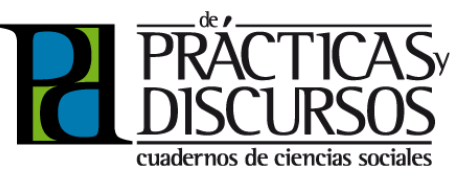

distintas plataformas virtuales, en este caso las páginas web de cada una de las organizaciones, y se puede pensar como una interacción fluida, dinámica y móvil donde los límites no son asunciones a priori, sino que se exploran en el curso de la etnografía con particular énfasis en la frontera entre "lo virtual y lo real" (De Sena y Lisdero, 2015: 84-85).

Según su página web, Techo es una organización que está presente en Latinoamérica que tiene como visión lograr una sociedad justa y sin pobreza, donde todas las personas tengan las oportunidades para desarrollar sus capacidades y puedan ejercer y gozar plenamente sus derechos. Dentro de sus objetivos se encuentra en primer lugar el fomento del desarrollo comunitario en asentamientos precarios. El desarrollo comunitario es considerado como eje transversal del trabajo de Techo en asentamientos precarios. En segundo lugar, se encuentra la promoción de la conciencia y acción social. El último objetivo es la incidencia en política, que promueva los cambios estructurales necesarios para que la pobreza disminuya rápidamente. Esto se logrará a través de la denuncia de la exclusión y vulneración de derechos dentro de los asentamientos; de la generación y difusión de información relevante sobre estos, para que sus problemas sean reconocidos por la sociedad y prioritarios en la agenda pública; y de la vinculación de los pobladores de asentamientos organizados con instituciones de gobierno.

En este último objetivo es donde se ubica su participación en la Ley de Regularización Dominial para la Integración Sociourbana, la cual llevó todo un proceso para poder tener los datos sobre el contexto habitacional que presenta el país a través de encuestas que formarían parte del Renabap, pero que tenía como meta la sanción de la ley.

En primera medida se relevaron todas las ciudades de más de 10 mil habitantes del país, con encuestados pagos por el gobierno nacional, que en la gran mayoría eran mismos pobladores de los barrios populares. Siempre el primer objetivo grande de esto fue la ley, y ahí se consiguió algo más intermedio, que fue la creación del Renabap, el Registro Nacional de Barrios Populares mediante el Decreto 358 en 2017, que ese decreto además de crear el registro, creaba el Certificado de Vivienda Nacional; y esa fue la primera herramienta que se tuvo desde las organizaciones y de los barrios para empezar a ser reconocidos oficialmente por el Estado nacional, y poder empezar a tramitar los servicios, que sirva como un 
De Prácticas y discursos

Universidad Nacional del Nordeste

Centro de Estudios Sociales

Año 8, Número 12, 2019, 0ctubre

ISSN 2250-6942
Organizaciones sociales del hăbitat y su influencia

en la formación de las leves y las políticas sociales

The social organizations of the habitat and their influence on the formation of laws and social policies

documento legal de reconocimiento de domicilio, etc. A nivel general, uno de los puntos más importantes y más urgentes que tiene la ley es que suspende los desalojos por cuatro años de todos los barrios que están dentro del registro nacional. (Entrevista organización social Techo 1, 2019: 8)

La discusión frente a esta ley es la figura de expropiación de terrenos por parte de la Agencia de Administración de Bienes del Estado y el contexto político en el que se aprueba cercano a las elecciones presidenciales 2019, teniendo en cuenta el rol que jugó el gobierno tanto para gestarla como para sancionarla de forma muy rápida. Estas críticas son observadas desde la Asociación Civil Madre Tierra, ubicada en Morón y compuesta por 22 integrantes, entre los que hay trabajadores sociales, arquitectos, contadores, abogados y otros profesionales que trabajan divididos en diferentes áreas internas para cubrir las necesidades de personas que están en situación de irregularidad en el dominio de sus tierras y están luchando para lograr condiciones de hábitat confortable. Según su página web, Madre Tierra trabaja desde 1985 para brindar información y asesoramiento en barrios en desarrollo y asentamientos que comienzan a ordenarse. También participa activamente con la sociedad para inducir politicamente acciones que lleven a la sanción de leyes referidas a la regularización dominial para que los ocupantes de la tierra sean sus legítimos dueños, el mejoramiento de la sustentabilidad ambiental y condiciones de habitabilidad dignas.

Fue todo tan rápido, tan poco consultado, tan nada concensuado, hasta secreto entre las cinco organizaciones que vienen sentándose en el Renabap que nos pareció muy extraño. Nosotros consideramos, porque tenemos 35 años de experiencia en regularización dominial, que la expropiación es el último recurso. Hay muchísimos otros instrumentos gratuitos donde el Estado no tiene que pagar nada para que los ocupantes puedan acceder a la regularización del dominio del lugar que ocupa. Esto es lo que hace que nos parezca muy raro que haya una ley que solo prevea eso que para nosotros es el último recurso. Ninguno de los sectores que estamos trabajando en el tema podemos decir que nos oponemos, lo que sí, tenemos la leve sospecha de que es un engaño electoral porque a cada familia que fue encuestada por el Renabap le ha llegado a su teléfono y le siguen llegan- 
De Prácticas y discursos

Universidad Nacional del Nordeste

Centro de Estudios Sociales

Año 8, Número 12, 2019, Octubre

ISSN 2250-6942

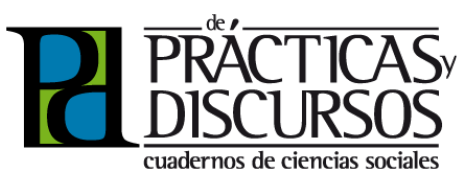

do propagandas donde le dicen que va a ser dueño de su lugar. Y es mentira que va a ser dueño de su lugar porque una expropiación es un proceso que lleva años. (Entrevista organización social Madre Tierra 2, 2019: 9)

Madre Tierra resalta la importancia de la Ley de Acceso Justo al Hábitat 14449 a escala provincial, que surge desde las experiencias de varias organizaciones con las que la asociación tiene contacto y fue presentada en la provincia de Buenos Aires a partir de la constatación de que hay un problema de desigualdad en la distribución y la gestión del territorio en el que se le ha entregado el territorio al mercado con sus lógicas de ganancia y especulación, lo que ha hecho que haya más tierra en pocas manos y menos posibilidad de tierra para las grandes mayorías. Según Madre Tierra, la Ley de Acceso Justo al Hábitat da vuelta esa dinámica.

La ley genera recursos para que los municipios puedan gestionar el territorio de manera equitativa, sustentable, sostenible, impulsiva, justa, productiva. Le da instrumentos a los municipios para, por un lado, gestionar el territorio, por otro lado, recuperar el recurso que a través de instrumentos que recuperan plusvalía, valorizaciones, recuperan fundamentalmente tierra que por ahí los propietarios de grandes predios no pueden urbanizar, entonces propone que el Estado urbanice junto con ellos. Entonces de esta manera recupera tierras, dinero, como para reinvertir en procesos sociales que tengan que ver con acceso de los sectores medios $y$ populares de un lugar donde vivir, construcciones, nuevos barrios, nuevas urbanizaciones, más infraestructuras, más equipamientos. Esta es la Ley de Acceso Justo al Hábitat. En esto es en lo que Madre Tierra junto con las organizaciones con las que luchó viene trabajando. (Entrevista organización social Madre Tierra 2, 2019: 11)

Aunque las dos organizaciones concuerdan en que esta ley es fundamental en materia de derecho a la vivienda, Techo-Argentina, al ser una organización joven, no llegó a participar en el proceso que fue el armado de la Ley de Acceso Justo al Hábitat. Si bien la utilizaron como marco legal para todo el trabajo que vienen realizando como organización, también surgieron críticas hacia la misma debido al posicionamiento que representa la ley en la actualidad. 
De Prácticas y discursos

Universidad Nacional del Nordeste

Centro de Estudios Sociales

Año 8, Número 12, 2019, Octubre

ISSN 2250-6942
Organizaciones sociales del hăbitat y su influencia

en la formación de las leves y las políticas sociales

The social organizations of the habitat and their influence on the formation of laws and social policies

Para mí, la 14449 es una ley excelente a nivel de lo que propone en la teoría para aplicación, siento que ha faltado un posicionamiento grande de la ley per se, y que los municipios realmente empiecen a laburar por ello, y que la provincia también le dé un impulso mucho más grande. $Y$ eso pasa porque o se fue perdiendo con el tiempo y fue un buen primer impulso en ese momento, y después como que fue diluyéndose un poquito y ahora es una herramienta que no tenemos que dejar morir. (Entrevista organización social Techo 1, 2019: 7)

\section{CONCLUSIÓN}

Teniendo en cuenta lo visto en la primera parte de este análisis sobre el capital, las políticas sociales y la cuestión social donde se afirma que los procesos de desigualdad y expulsión generados en la estructuración de una sociedad basada en la mercantilización de la vida provocan quiebres conflictuales que deben ser subsanados sistémicamente, aparece el rol de las organizaciones sociales que se involucraron en la gestión del hábitat y la acción territorial. En este recorrido se pudo definir qué son las organizaciones sociales, qué ventajas y desventajas presentan frente al Estado, pero se debe volver a remarcar que en ningún caso pueden reemplazar al Estado. Sí brindarles herramientas para que se puedan crear políticas sociales cercanas a su experiencia, como sucede en la formación de las leyes que existen a escala provincial y nacional en la Argentina, donde las organizaciones de la sociedad civil tienen una participación clave.

Al retomar lo expuesto en el análisis, en principio las dos organizaciones concuerdan en que la Ley 14449 de Acceso Justo al Hábitat representa un logro desde las organizaciones sociales para incidir en política, ya que desde su lugar se gestó y se promovió para que se sancione la ley y enmarca los principios fundamentales en materia de derecho a la ciudad. Pero no hay que dejar de observar la crítica que realiza Techo respecto a la ley, debido a que si bien es un logro como ley desde toda la teoria que tiene, si no se aplica como corresponde en los municipios deja de tener sentido el esfuerzo que se realizó para tenerla como marco legal. Por otro lado, sobre la Ley 27453 de Regularización Dominial para la Integración Socio Urbana, el panorama es otro, ya que no hay un acuerdo entre las dos organizaciones debido a que Madre 
Tierra tiene una cierta desconfianza tanto al relevamiento que se llevó a cabo en primera instancia como a la ley en sí, resaltando el hecho de que propone la expropiación de la tierra por parte del Estado nacional y a los impulsores de la misma, teniendo en cuenta que se gestó en 2015 con la asunción del nuevo gobierno nacional de la Alianza Cambiemos. Por otro lado, Techo-Argentina es considerada una de las impulsoras de esta ley y es desde esta organización que se toma la metodología para realizar el relevamiento de barrios populares, por lo tanto, tiene una visión positiva sobre la misma.

\section{BIBLIOGRAFIA}

ARGENTINA. MINISTERIO DE INFRAESTRUCTURA. SUBSECRETARÍA SOCIAL DE TIERRAS, URBANISMO Y VIVIENDA, PROVINCIA DE BUENOS AIRES. (2015). Ley 14449. Informe de gestión.

BUSTOS, W. (2015). Ley 14.449 de Acceso Justo al Hábitat. Su implementación en los partidos de la Región Metropolitana de Buenos Aires. Universidad Nacional de General Sarmiento, Observatorio del Conurbano Bonaerense. Disponible en: http:// observatorioconurbano.ungs.edu.ar/? $p=6379$

CENTRO DE ESTUDIOS LEGALES Y SOCIALES (2017). Herramientas para aplicar la ley de acceso justo al hábitat. Buenos Aires, Argentina.

CHAHBENDERIAN, F. (2018) Estado, cuestión social y regulación sensible: Aportes desde las políticas sociales. Controversias y Concurrencias Latinoamericanas, (17)10, 17-35. Extraído de: http://bit.ly/2DNScfE

DE PIERO, S. (2005). Organizaciones de la sociedad civil. Tensiones de una agenda en construcción. Buenos Aires: Paidós.

DE SENA, A. (2011). Promoción de microemprendimientos y políticas sociales: ¿Universalidad, focalización o masividad?, Una discusión no acabada. Pensamiento Plural, 37-63.

DI VIRGILIO, M. (2012). Participación social y organizaciones 
sociales en la implementación de políticas orientadas a la producción social del hábitat en el área metropolitana de Buenos Aires, Argentina. Revista SAAP, 6(1). Recuperado de: https://bit. Iy/2AMT8zF

GUEVARA, T. ET AL. (2018). Integración urbana y políticas públicas: el caso del Registro Nacional de barrios populares de Argentina: Decreto No 358/2017. Oculm Ensaios, Revista de Arquitectura e Urbanismo. Extraído de http://dx.doi. org/10.24220/2318-0919v15n3a4183

GOBIERNO DE LA PROVINCIA DE BUENOS AIRES (2018). Boletín Oficial. Suplemento (p. 10). Buenos Aires, Argentina. Disponible en https://www.boletinoficial.gba.gob.ar/sections/8458/ view\#page $=10$

MAGLIERI, G. (2003). Dirección de organizaciones sociales ( $1^{\text {a }}$ ed) (p. 307). Buenos Aires: Ugerman. Propuestas. ISBN 9789879468081.

MONCAYO, M. (2016). La etnografía virtual como método de investigación en el diagnóstico de la comunicación corporativa. Estrategas, investigación en la comunicación, 3, 114-125. Recuperado de: http://marketing.udla.edu.ec/ojs/index.php/estrategas/article/view/104

ORTIZ FLORES, E (2010). Derecho a la ciudad, producción social y gestión participativa del hábitat. La promoción de iniciativas comunitarias incluyentes en la Ciudad de México. Hábitat y Sociedad, 1, 55-70. Disponible en: www.habitatysociedad.us.es

SCRIBANO, A Y DE SENA, A. (2014). Consumo Compensatorio: ¿Una nueva forma de construir sensibilidades desde el Estado? Revista Latinoamericana de Estudios sobre Cuerpos, Emociones y Sociedad, 2, 65-82. Disponible en: http://www.relaces.com. ar/index.php/relaces/article/viewArticle/335 